\title{
Band structure and optical properties of opal photonic crystals
}

\author{
E. Pavarini, L.C. Andreani, C. Soci, M. Galli, and F. Marabelli \\ Istituto Nazionale per la Fisica della Materia and Dipartimento di Fisica "A. Volta", \\ Università di Pavia, Via Bassi 6, 27100 Pavia, Italy \\ D. Comoretto \\ Istituto Nazionale per la Fisica della Materia and Dipartimento di Chimica e Chimica Industriale, \\ Università di Genova, via Dodecaneso 31, 16146 Genova, Italy
}

(Dated: July 3, 2021)

\begin{abstract}
A theoretical approach for the interpretation of reflectance spectra of opal photonic crystals with fcc structure and (111) surface orientation is presented. It is based on the calculation of photonic bands and density of states corresponding to a specified angle of incidence in air. The results yield a clear distinction between diffraction in the direction of light propagation by (111) family planes (leading to the formation of a stop band) and diffraction in other directions by higher-order planes (corresponding to the excitation of photonic modes in the crystal). Reflectance measurements on artificial opals made of self-assembled polystyrene spheres are analyzed according to the theoretical scheme and give evidence of diffraction by higher-order crystalline planes in the photonic structure.

PACS numbers: 42.70.Qs, 41.20.Jb, 78.40.-q
\end{abstract}

\section{INTRODUCTION}

Three-dimensional photonic crystals are intensively studied with the goal of achieving control of light propagation and, especially, a complete photonic band gap in

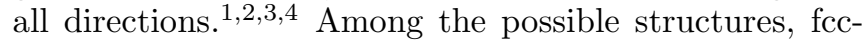
based systems like opals and inverse opals are of great interest as they can be produced with bottom-up approaches based on casting from colloidal solutions, possibly followed by infiltration and template removal $\stackrel{5,6.7 .8}{=}$ The direct opal structure (e.g., polystyrene or silica spheres in air) has weak effects on the total photonic density of states (DOS) but does produce a well-defined stop band in the direction of light propagation. If the structure crystallizes in an fcc lattice with (111) surface orientation, as is usually the case, normal-incidence reflectance or transmittance probe the photonic gap in the $\Gamma L$ direction of the fcc Brillouin zone. Instead, the inverse opal structure (e.g., air spheres in $\mathrm{TiO}_{2}$ or in silicon) produces a strong effect on the photonic DOS and may even result in a complete photonic band gap if the dielectric constant and the filling fraction of the infiltrated material are large enough. ${ }^{9.10}$

The optical properties of fcc colloidal photonic crystals have been the subject of numerous experimental 11.12 .13 .14 .15 .16 .17 .18 .19 .20 .21 .22 .23 .24 .25 .26 .27 .28 .29 .30 and theoretical9.10.20.34.35.36.37.38.39 studies. Many experimental investigations are concerned with properties of the lowest-order gap, in particular the attenuation studied by normal-incidence transmission ${ }^{13.15}$ and the evolution of the stop band in reflectance/transmittance spectra at varying angles of incidence 14.16.17.20.24.25.32 and as a function of polarization ${ }^{30}$ Detailed studies of the high-energy region at normal incidence have recently appeared ${ }^{33}$ The role of disorder in Bragg scattering and gap formation has been addressed with measurements on multiple-domain ${ }^{21,29}$ and single-domain crystals ${ }^{22,28}$
Bragg diffraction has also been studied in a transmission geometry by Kossel line analysis. ${ }^{12}$ The change in effective refractive index close to the first-order gap and in connection with flat bands at high energies has also been studied ${ }^{18.31}$ Laser dyes in opal photonic crystals show effects related to modified photonic density of states in emission spectra ${ }^{19,23,27}$ and to interaction of photonic modes with excitons $\underline{40}$ On the theoretical side, the formation of a complete band gap in the inverse opal structure ${ }^{9.10}$ and its fragility against disorder ${ }^{35}$ are now well understood. The interplay between photonic bands and reflection/transmission properties has been addressed both with calculations of optical spectra 20.29 .34 and superprism effects ${ }^{37}$ as well as with symmetry analysis $\frac{38}{3}$ More recent works focus on the effects of stacking faults on the optical properties $\frac{36.39}{69}$

A question of particular interest is the role of diffraction from lattice planes other than the (111) family in connection with the optical properties at energies higher than the first stop band along the $\Gamma L$ direction. The analysis of Kossel lines in transmission has led to a determination of the photonic gap positions for the two lowest band gaps 12 : the results for the band edges are reported as a function of incidence angle along the LW direction of the fcc Brillouin zone. This interpretation has been 31.32 .33 oned in Ref. 34 on the basis of calculations of transmission spectra and projected photonic bands for given values of the wavevector $\mathbf{k}_{\|}$parallel to the crystal surface. Angle-resolved reflectance measurements give evidence of multiple diffraction peaks with small splittings at large values of the angle of incidence, $\stackrel{24,25.26 .32}{ }$ which have been interpreted as arising from multiple Braggwave coupling $24,25,32$ or photonic band branching, ${ }^{26}$ both concepts being related to an anticrossing of photonic levels close to the $\mathrm{U}$ or $\mathrm{W}$ points of the Brillouin zone. Related observations but a different interpretation is given in Ref 31, in which additional peaks in reflectance (or 


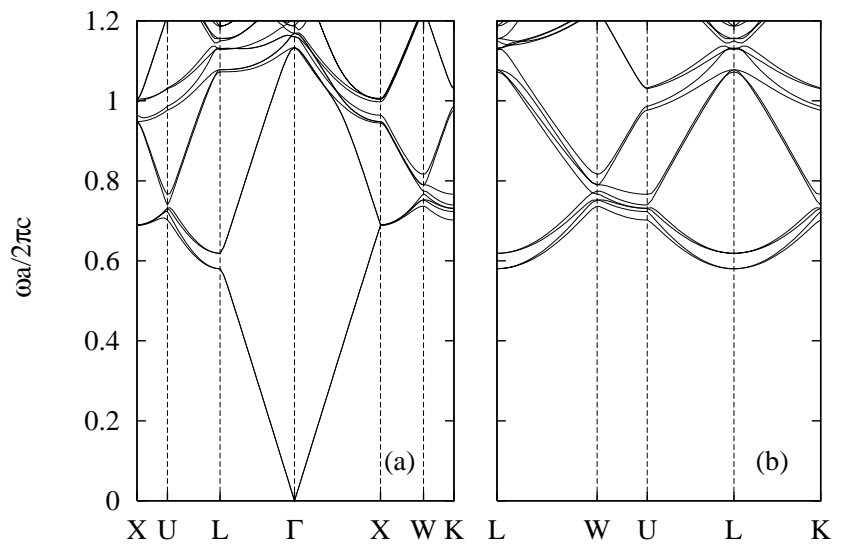

FIG. 1: Energy bands for an opal photonic crystal (fcc lattice of close-packed dielectric spheres with $\epsilon_{1}=2.53$ in air): (a) along symmetry directions in the whole Brillouin zone, (b) along symmetry directions on the hexagonal face of the Brillouin zone.

dips in transmittance) at near-normal incidence are attributed to a large effective refractive index arising from flat bands. The main issue in the interpretation of all these results is that spectral features in optical measurements cannot be unambiguously associated with the presence of a photonic gap and/or with diffraction. For reflectance measurements, in particular, a spectral feature may follow from the existence of a photonic gap (as is the case for the lowest stop band at near-normal incidence) but also from the excitation of a photonic mode in the crystal: establishing a clear distinction between the two effects is the main purpose of the present paper.

In this work we present a theoretical approach to the optical properties of 3D photonic crystals which yields further insight in the phenomenon of diffraction from different families of lattice planes in relation to the presence of photonic gaps or photonic bands. We calculate the photonic bands and density of states corresponding to a fixed external angle of incidence: the DOS is decreased when a photonic gap along the propagation direction occurs, while it increases and shows resonant structures when diffraction in directions other than that of specular reflection takes place. Calculations of reflection and diffraction spectra show that peaks in the DOS correspond to the onset of diffraction, as well as to additional reflectance structures at large values of the angle of incidence. Reflectance measurements on opal photonic crystals are presented and interpreted with the above concepts. In particular, weak features at large angles of incidence give experimental evidence for the excitation of photonic modes in the crystal, i.e., for diffraction from planes other than those of the (111) family.

The results of this work are related to the surface reflectance technique for measuring the photonic band

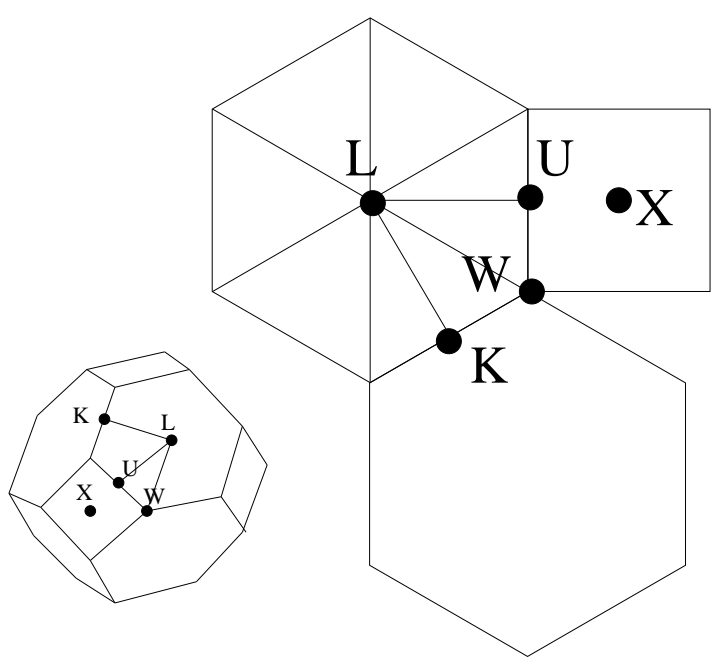

FIG. 2: Symmetry points in the Brillouin zone for a fcc lattice.

dispersion. This approach was first applied to photonic crystal waveguides, where it led to a determination of the dispersion of photonic modes above the light line via the observation of resonant structure in reflectance and of their dispersion with incidence angle. ${ }^{41.42 .43 .44}$ The same technique of variable-angle reflectance from the crystal surface can be applied to the case of macroporous silicon, which has a 2D photonic structure and can be considered as homogeneous in the direction of the pores, and in this case it yields the dispersion of photonic bands in the 2D plane. ${ }^{45}$ The present work shows that, in the case of photonic crystals with refractive index modulation in $3 \mathrm{D}$, Bragg diffraction off the direction of light propagation also produces spectral structures in reflectance, whose angular evolution is related to the photonic dispersion in a plane parallel to the crystal surface. Preliminary results have been recently presented 46

The rest of this paper is organized as follows. In Sec.II we present the theoretical approach, namely the calculation of photonic DOS and photonic bands corresponding to a specified angle of incidence. In Sec.III we describe reflectance experiments on opal photonic crystals and compare the theoretical predictions with the optical spectra. In Section IV the findings of this work are summarized.

\section{PHOTONIC BANDS AND DENSITY OF STATES}

We calculate the photonic bands starting from the second-order wave equation for the magnetic field

$$
\nabla \times\left[\frac{1}{\varepsilon(\mathbf{r})} \nabla \times \mathbf{H}\right]=\frac{\omega^{2}}{c^{2}} \mathbf{H} .
$$



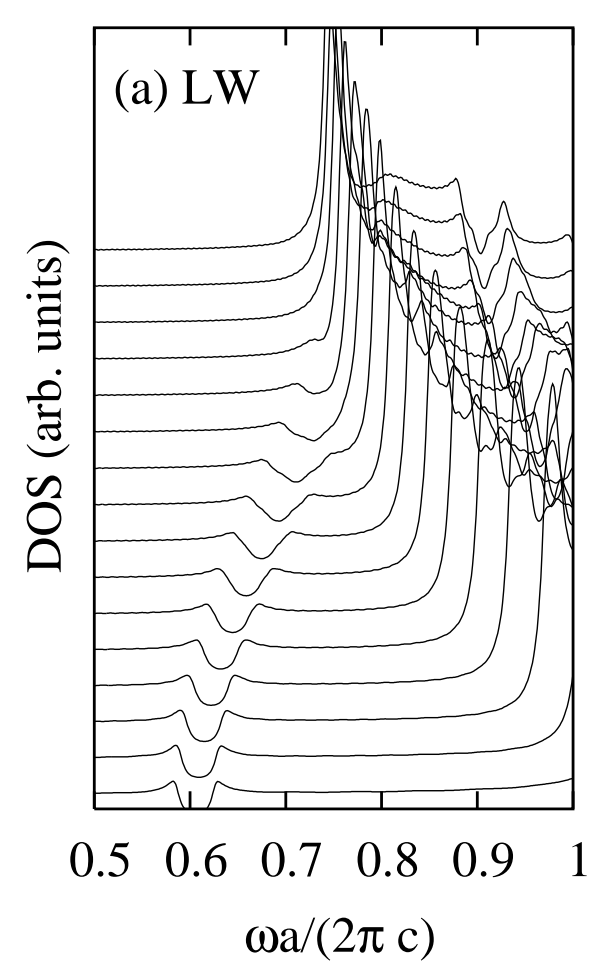
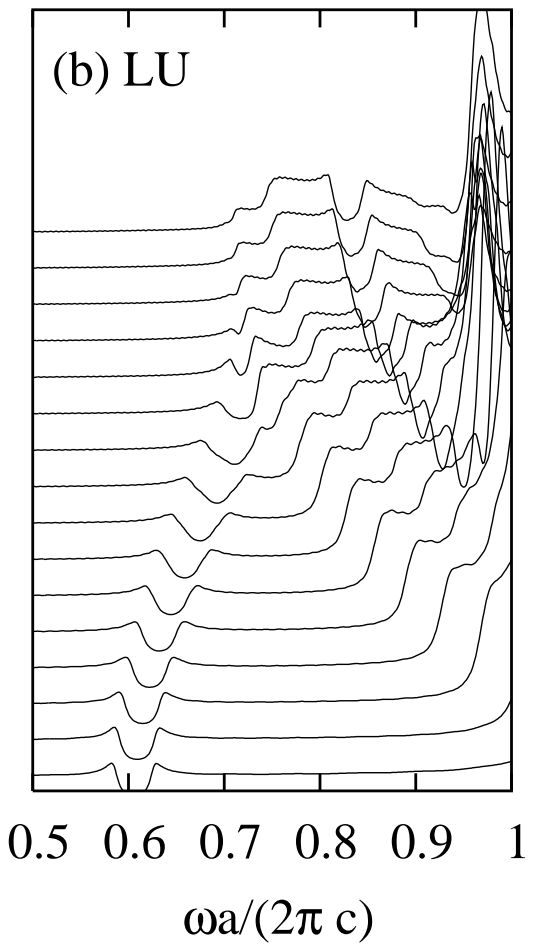

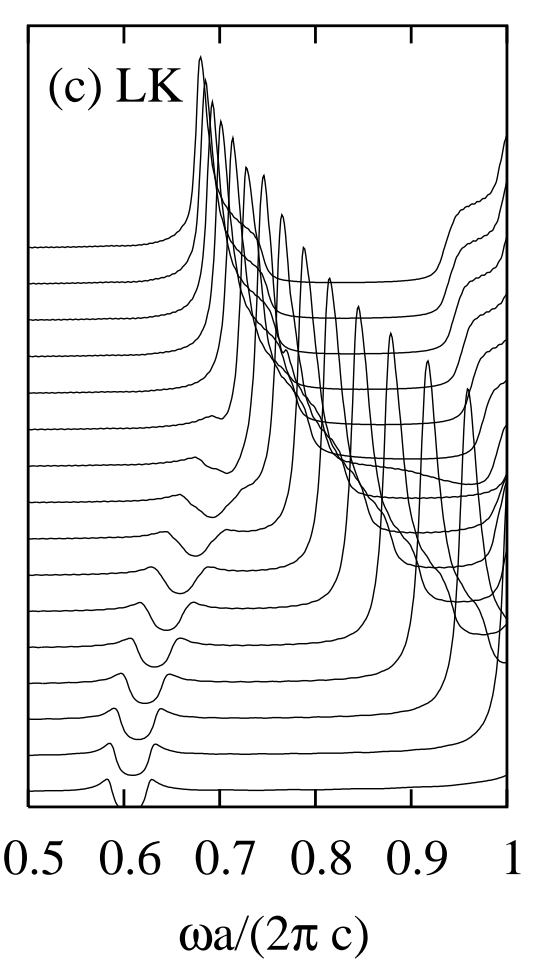

FIG. 3: Reduced density of states for several values of the incidence angle from $\theta=5^{\circ}$ (bottom curve) to $\theta=80^{\circ}$ (top curve) in steps of $5^{\circ}$. The three figures correspond to different values of the azimuthal angle $\phi$ : (a) $\phi=0^{\circ}+2 m \pi / 6(m \in \mathcal{Z})$, or LW direction; (b) $\phi=30^{\circ}+2 m \pi / 3$, or LU direction; (c) $\phi=-30^{\circ}+2 m \pi / 3$, or LK direction.

We adopt the plane-wave expansion technique to transform Eq. (1) into an eigenvalue problem, and calculate the inverse dielectric constant by using the procedure of Ho, Chan and Soukoulis $\frac{47}{4 n}$ Fig. 1 we show the photonic bands for an opal with dielectric constants $\epsilon_{1}=2.53$ (spheres) $)^{48.49}$ and $\epsilon_{2}=1$ (voids), i.e., polystyrene spheres in air. The fcc Brillouin zone with high symmetry points is shown in Fig. 2. The bands are shown in dimensionless units $\omega a /(2 \pi c)$, where $a$ is the lattice constant, along the symmetry lines of the full fcc Brillouin zone (Fig. 1a) as well as along symmetry lines on the (111) hexagonal face (Fig. 1b): the latter will be useful for comparing with density of states and reflectance spectra at a finite angle of incidence.

The photonic bands in Fig. 1 do not exhibit any complete gap in the full Brillouin zone (BZ). However, partial gaps do appear along some symmetry lines of the BZ; among these, the lowest energy gap opens along $\Gamma \mathrm{L}$ around $\omega a /(2 \pi c) \sim 0.6$. Partial gaps behave as stop bands in reflectance spectra when they occur in the direction of propagation. Photonic modes along the propagation direction also contribute to the optical properties and may give rise to additional features in reflectance, as discussed in the rest of this Section.

In order to distinguish reflectance maxima due to stop bands from features which can instead be associated to photonic modes, we need first of all to calculate the photonic band structure and the density of states (DOS) along the direction of propagation, i.e., for a specified value of the angle of incidence in air. In the following we will refer to these bands and DOS as reduced bands and reduced DOS, respectively. The surface of a fcc colloidal crystal is known to be a (111) crystallographic plane, therefore angle-resolved reflectivity experiments are usually performed as a function of the angle of incidence with respect to a (111) surface. We adopt the same convention in our calculations and define a reference frame as follows: $\hat{z}$ is the $[1,1,1]$ or $\Gamma \mathrm{L}$ direction, $\hat{x}$ is the $[1,0,-1]$ (a $\mathrm{LW}$ ) direction, and $y$ is the $[1,-2,1]$ (a LK) direction. We can therefore express the momentum of an incident photon as follows: $\mathbf{k}^{i}=\frac{\omega}{c}(\sin \theta \cos \phi, \sin \theta \sin \phi, \cos \theta)$, where $\hbar \omega$ is the energy of the incident photon. With our choice of axes, $\theta=0^{\circ}$ corresponds to normal incidence ( $\Gamma \mathrm{L}$ direction) and the azimuthal angle $\phi$ is measured from the $\mathrm{LW}$ direction. We stress that $\theta$ is the angle of incidence in air (which differs from the angle of propagation inside the opal,, 37 the latter being dependent on the effective refractive index and on the presence of additional propagating modes when diffraction inside the material occurs).

The reduced bands and state densities are obtained from the full band structure (Fig. 1) by using conservations laws: (i) parallel momentum conservation modulo a reciprocal lattice vector, and (ii) energy conservation. For the photonic bands, these two conditions may be 
written as

$$
\begin{aligned}
\mathbf{k}_{\|}+\mathbf{G}_{\|} & =\frac{\omega}{c}(\sin \theta \cos \phi, \sin \theta \sin \phi, 0), \\
\epsilon_{n}\left(\mathbf{k}_{\|}, \mathbf{k}_{z}\right) & =\hbar \omega,
\end{aligned}
$$

where $\mathbf{k}_{\|} \equiv \mathbf{k}_{\|}^{i}$ is the component of $\mathbf{k}$ parallel to the (111) surface, $\mathbf{G}_{\|}$is the parallel component of any reciprocal lattice vector $\mathbf{G}, \mathbf{k}_{z}$ is the component of $\mathbf{k}$ parallel to $\Gamma \mathrm{L}$, and $\epsilon_{n}\left(\mathbf{k}_{\|}, \mathbf{k}_{z}\right)$ is the $n$-th energy band of a photon with momentum $\mathbf{k}$. We point out that $\mathbf{k}_{\|}$is fixed by the experimental configuration. On the contrary $\mathbf{k}_{z}$ and the reduced bands are obtained from the solutions of the system given by Eqs. (2), (3), which are found by a selfconsistent procedure. The reduced DOS is obtained from the reduced bands as follows:

$$
N(\hbar \omega)=\sum_{n, \mathbf{k}_{z} \geq 0} \delta\left(\epsilon_{n}\left(\mathbf{k}_{\|}, \mathbf{k}_{z}\right)-\hbar \omega\right),
$$

where $\mathbf{k}_{\|}$is defined by Eq. (2). The above $N(\hbar \omega)$ is a one-dimensional density of states, because it is summed only over $\mathbf{k}_{z}{ }^{50}$ The DOS obtained in this way (convoluted with a Lorentzian of width comparable to a typical experimental resolution $\Delta \omega=10^{-2} \cdot 2 \pi c / a$ and averaged over photon polarizations) is shown in Fig. 3 for several values of $\theta$ and some values of $\phi$ (directions LW, LU and LK) 51

Let us analyze first the results for $\theta=0^{\circ}$. In this case the solution of Eqs. (2) and (3) can be found very simply because $\mathbf{k}_{\|}=0$, and it yields the photonic bands along the $\Gamma \mathrm{L}$ direction. Thus we can directly compare the $\theta=$ $0^{\circ}$ DOS in Fig. 3 with the bands along $\Gamma \mathrm{L}$ in Fig. 1a. The lowest energy gap shown in Fig. 1 around $\omega a /(2 \pi c)=0.6$ appears clearly in the reduced DOS at the same values of the energy. It arises from diffraction in the backwards direction by planes of the (111) family. The photonic bands at energies above the gap in Fig. 1a correspond to a photon momentum $\mathbf{k}$ outside the first Brillouin zone; the bands are folded into the first BZ by subtracting from $\mathbf{k}$ a reciprocal lattice vector $(2 \pi / a)(1,1,1)$. These folded bands give rise to a finite DOS above the band gap in Fig. 3.

The reduced DOS reflects, of course, the energy dispersion of the photonic bands along the propagation direction. The latter is almost linear at low frequencies, and thus gives rise to a nearly constant one-dimensional DOS. For the case shown in Fig. 1a, the bands along $\mathrm{CL}$ just above the gap at $\omega a /(2 \pi c)=0.6$ exhibit also an almost linear dispersion, thus the corresponding DOS in Fig. 3 remains flat above the gap. However, in general, the folded bands may exhibit a non-linear dispersion and can also show critical points (maxima, minima, or saddle points). In three dimensions, a critical point results in a divergence of the slope of the DOS (van Hove singularity). In the present one-dimensional case the DOS itself diverges at a van Hove singularity. The very flat bands at energy around $\omega a /(2 \pi c)=1.1$ (the fifth and sixth bands of the photonic structure) show one of such critical points at L; as a consequence, the corresponding reduced DOS at normal incidence exhibits a Van Hove singularity around $\omega a /(2 \pi c)=1.1$. This critical point is out of the scale of Fig. 3 (it is shown in Fig. 4 below); however, as we discuss in next paragraph, it moves to lower energies when moving from normal incidence.

The complex, nonlinear dispersion of the photonic bands results from the mixing of plane waves with different reciprocal lattice vectors (in directions other than the (111) one) in the photonic Bloch mode, thus it implies the occurrence of diffraction in directions other than that of beam propagation. Notice that this phenomenon can occur at any angle of incidence, even for $\theta=0^{\circ}$, when the conservation of parallel crystal momentum expressed by $\mathrm{Eq}(2)$ can be satisfied with $\mathbf{G}_{\|}=0$. For energies below $\omega a /(2 \pi c)=1.5$, the photonic bands are determined by reciprocal lattice vectors of lower modulus, the relevant ones being (000), the eight equivalent $\{111\}$ and the six equivalent $\{200\}$ ones. Thus the directions of diffraction out of the propagation direction (forward or backward) are determined by planes of equivalent $\{111\}$ families that are not parallel to the (111) crystal surface, as well as by $\{200\}$ families. At near-normal incidence, diffraction directions just above the diffraction cutoff are

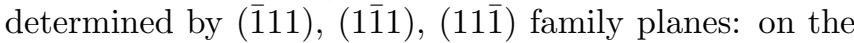
other hand, $\{200\}$ families become important at large angles of incidence, as discussed below. We conclude that the presence of a van Hove singularity in the reduced DOS marks the onset of diffraction from lattice planes other than those of the (111) family.

Let us analyze now the case of finite incidence angles. Figure 3 shows how the stop band depend strongly on the incidence angle, by moving to higher energies with increasing $\theta$. On the other hand, it depends only weakly on the azimuthal angle $\phi$. Figure 3 shows also the peaks associated to the Van Hove singularity. The latter is at $\omega a /(2 \pi c)=1.1$ at normal incidence, but it moves at lower energies as $\theta$ increases. This is shown clearly in Figs. $3 \mathrm{a}$ and $3 \mathrm{c}$, in which this singularity appears as a strong peak in the DOS: this peak merges with the gap around $\theta=70^{\circ}$ and $60^{\circ}$, respectively. Notice that since both the gap and the van Hove singularities occur on the hexagonal face of the fcc Brillouin zone, their angular dependence is related to the wavevector dependence of the bands shown in Fig. 1b. In particular, along the LW orientation, the merging of the van Hove singularity with the gap occurs close to the $\mathrm{W}$ point (see Fig. 1b), where multiple Bragg scattering from $\{111\}$ and $\{200\}$ family planes occurs. We point out that the position of the Van Hove singularity depends strongly on $\phi$, as we may see by comparing Figs. 3a,c with Fig. 3b. In particular, for $\phi=30^{\circ}$ (LU direction) the Van Hove singularity remains almost outside the energy window.

Photonic modes give rise to several other features in the reduced DOS. All these features are associated with folded bands and exhibit a similar behavior as a function of $\theta$. This can be seen clearly in Fig. 3b. In this case the van Hove singularity remains very high in energy for any 
(a)
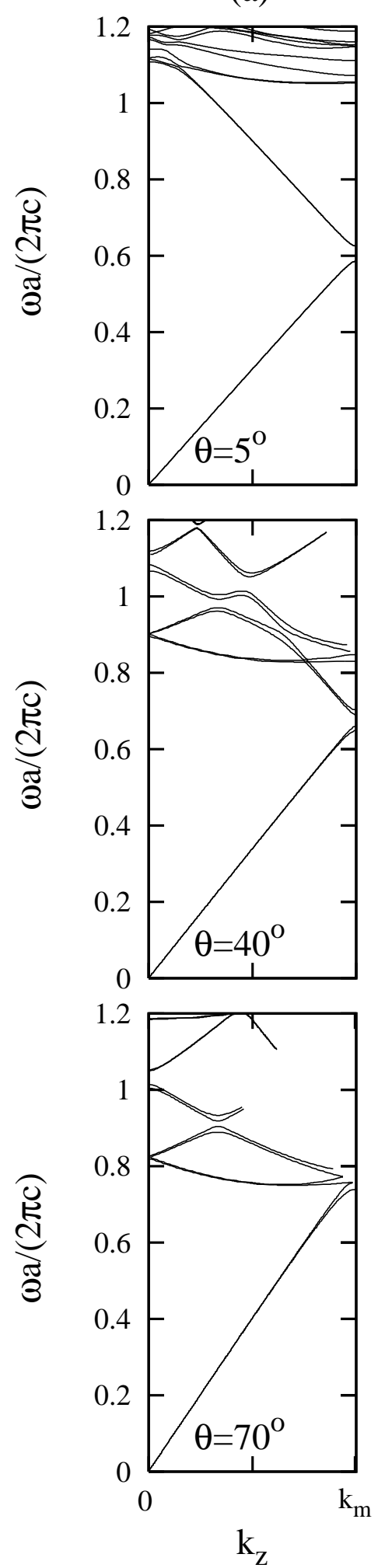

(b)
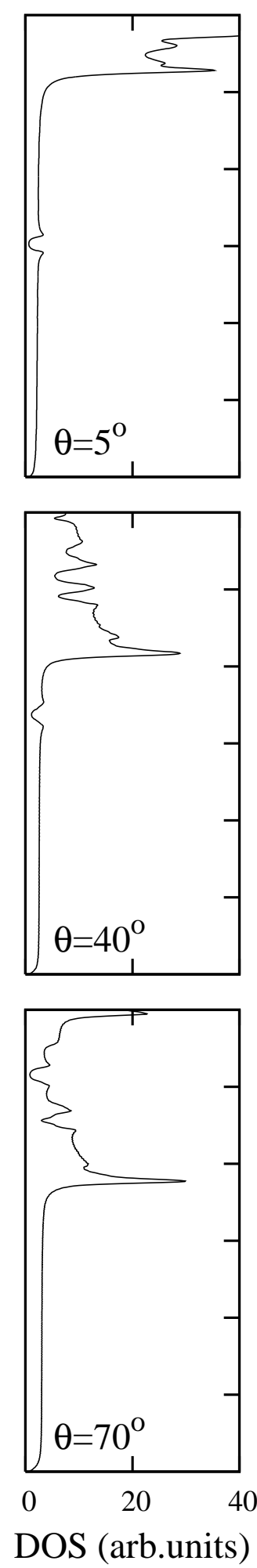

(c)
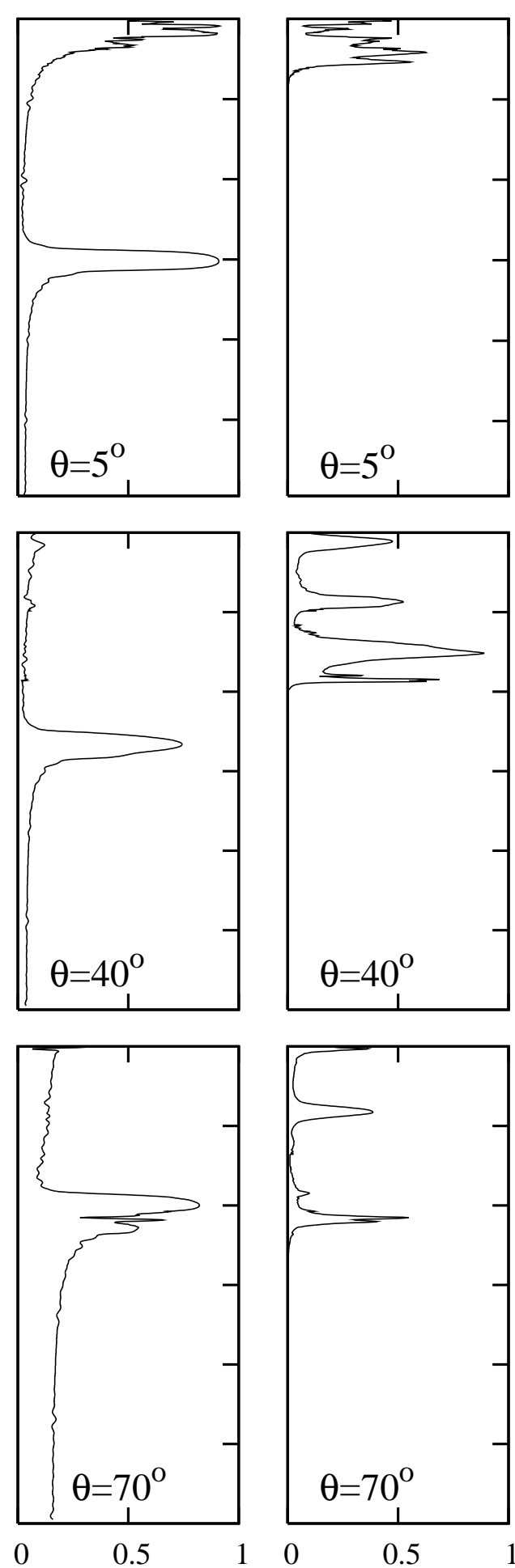

Reflectance (d)
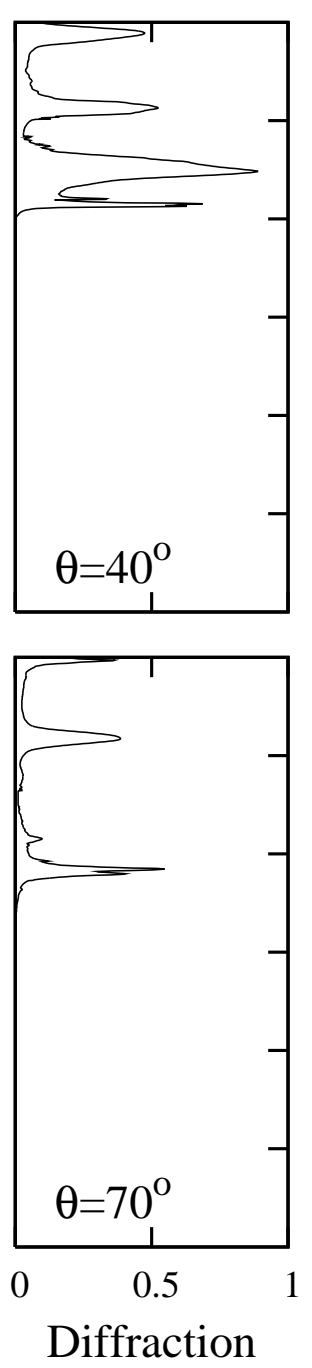

FIG. 4: (a) Reduced bands, (b) reduced DOS, (c) reflectance and (d) diffraction spectra for $\phi=0^{\circ}$ (LW direction) and three values $\theta=5^{\circ}, 40^{\circ} 70^{\circ}$ of the angle of incidence in air. The bands in (a) are plotted in the interval $\left(0, k_{m}\right)$, where $k_{m} \equiv \sqrt{3} \pi / a$ is the $\Gamma$-L distance. In the limit $\theta=0$ the point $k_{m}$ is identical to L. The bands are displayed only for $k_{z}$ inside the first Brillouin zone.

value of $\theta$. The steps seen in the DOS are instead associ- $\quad$ ated with bands which have an almost linear dispersion; 
a step indicates that a new band is becoming accessible. Furthermore, Fig. 3b shows also a second photonic gap at higher energies, which is present but much weaker for $\phi=0^{\circ}$ (LW direction, Fig. 3a), and is totally absent for $\phi=-30^{\circ}$ (LK direction, Fig. 3c).

In order to better clarify the $\theta$-dependence of the stop bands and of the van Hove singularities, in Fig. 4a,b we show the reduced bands and DOS in more detail. The bands are shown as a function of $\mathbf{k}_{z}$ and for three values of $\theta$. We focus on the LW direction $\left(\phi=0^{\circ}\right)$, but examination of the results for other orientations leads to similar conclusions. We notice that, since $\mathbf{k}_{z}$ is defined only inside the first BZ, its maximum length becomes shorter than $\sqrt{3} \pi / a$ for large $\theta$ and large frequencies (Fig. 4a for $\theta=70^{\circ}$ ), due to the truncated shape of the BZ in Fig. 2: this occurs when $\mathbf{k}_{\|}+\mathbf{G}_{\|}$is outside the hexagonal face containing the L point.

For near-normal incidence, the bands along the direction of propagation $\left(\theta=5^{\circ}\right)$ are simply the photonic bands along $\Gamma \mathrm{L}$, with the gap around $\omega a /(2 \pi c) \sim 0.6$ and the flat bands around $\omega a /(2 \pi c) \sim 1.1$. The panels in Fig. 4a show that, when $\theta$ increases, the gap moves to higher energy, while the flat bands and the associated van Hove singularity move downwards to lower energy. Eventually the gap and the flat bands overlap for $\theta \sim 70^{\circ}$ and $\omega a /(2 \pi c) \simeq 0.75$, corresponding to the $\mathrm{W}$ point of the fcc Brillouin zone (see Fig. 1b). These features are precisely reflected in the DOS, as we may see in the panels of Fig. 4b. In Fig. 4c,d we show reflectance and diffraction spectra for the same values of the angle of incidence, calculated by the scattering-matrix method ${ }^{52.53}$ Diffraction (D) spectra are obtained as $D=1-R-T$ in terms of reflectance $(\mathrm{R})$ and transmittance $(\mathrm{T})$. The stop band appears clearly in reflectance spectra. The onset of diffraction is around $\omega a /(2 \pi c)=1.1$ for $\theta=5^{\circ}$, $\omega a /(2 \pi c)=0.82$ for $\theta=40^{\circ}$ and $\omega a /(2 \pi c)=0.75$ for $\theta=70^{\circ}$. The first peak in diffraction spectra correlates very clearly with the peak in the reduced DOS (Fig. 4b) arising in turn from the flat bands and the Van Hove singularity (Fig. 4a).

We notice that other diffraction peaks and van Hove singularities are present in Fig.4b,d for frequencies above the diffraction cutoff. We do not pursue an analysis of these additional features, since they have no well-resolved counterpart in either theoretical reflectance spectra (Fig.4c) or in the experimental ones (see next Section), and also because the approximation made in the theoretical calculation becomes less accurate on increasing the energy 53 We emphasize that diffraction features become evident in reflectance spectra at high values of the angle of incidence, as clearly seen for $\theta=70^{\circ}$ in Fig.4c and discussed below in connection with the experimental data.

Thus the analysis of the reduced bands and DOS corresponding to a specified value of the angle of incidence leads to a clear distinction between diffraction effects (high DOS regions) from gaps or pseudogaps (zero or low DOS). In addition, critical points may give rise to van
Hove singularities which strongly enhance the DOS in the associated spectral region. The presence of a van Hove singularity marks the excitation of a photonic mode in the crystal and corresponds to the occurrence of diffraction in directions other than that of propagation. Van Hove singularities appear clearly in diffraction spectra and become evident also in reflection spectra at large values of the angle of incidence, i.e. close to the $\mathrm{W}$ point, where Bragg scattering is determined by planes of both $\{111\}$ and $\{200\}$ families. These conclusions are important for interpreting reflection experiments on polystyrene opals, as discussed in the next Section.

\section{OPTICAL SPECTRA}

\section{A. Experimental}

Bulk polystyrene opals have been grown by monodisperse sphere suspensions in water (Duke Scientific, diameter 222, 260, 300, 340, $426 \mathrm{~nm}$, refractive index 1.59) $\stackrel{48.49}{ }$ Small vessels with area of about one squared centimeter are obtained by sealing on a glass window polystyrene or silicone tubes. These vessels are filled with the sphere suspension and placed in a wet environment to reduce the evaporation rate. The opals have a meniscus shape and a glossy surface showing bright colored reflections.

Scanning Electron Microscopy (SEM) of a $222 \mathrm{~nm}$ opal surface has been performed with a Leo Stereoscan 440 (LEO Electron Microscopy Ltd) electronic microscope. The surface is very flat on a large scale (tens of microns) and shows a regular arrangement of the spheres in a triangular lattice as expected for their three-dimensional packing in a fcc lattice when observed along the (111) direction. Figure 5 shows a SEM image of $18 \times 12(\mu \mathrm{m})^{2}$ opal surface. This image can be considered as representative of the most disordered part of the opal surface investigated by the optical measurements. This morphology is very different from that of opal films we previously investigated, which were characterized by ordered domains of spheres of a few square microns areas with both triangular and square lattices, tilted with respect to each other and separated by disordered zones or deep dislocations ${ }^{54.55}$ For the present bulk opal samples, no square lattices are observed and the surface appears as a large triangular lattice of spheres with only a few defects.

Unpolarized variable-angle specular reflectance has been measured in the $0.4-4 \mathrm{eV}$ spectral range by means of a Fourier-Transform spectrophotometer Bruker IFS66 with $8 \mathrm{~cm}^{-1}$ spectral resolution. The light of a Xe arclamp was collimated and then focused to a spot of $100 \mu \mathrm{m}$ diameter on the sample surface. The sample was placed on a home-made $\theta-2 \theta$ goniometer, that allows the incidence angle to be varied between 5 and 80 degrees, with an angular resolution of $1^{\circ}$ determined by the angular divergence of the incident light cone. Sample visualiza- 


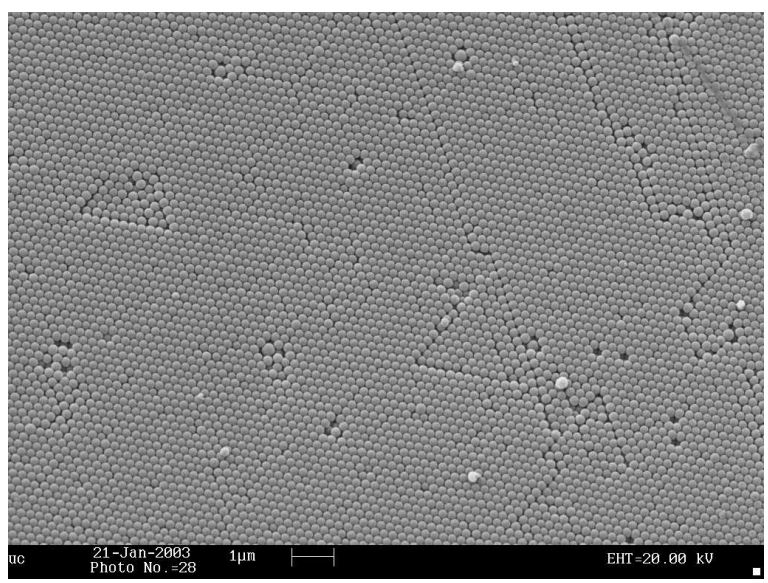

FIG. 5: SEM Image of the opal surface $\left(18 \times 22 \mu \mathrm{m}^{2}\right)$.

tion and precise allocation of the light spot on the surface was achieved by an optical microscope mounted on the goniometer. An Ag mirror was used as a reference. The overall error bar of the experimental set-up on the absolute value of the reflectance is within $2 \%$.

Particular care has been taken in the alignment of the sample. Since the top quality surface of the investigated sample is located close to one of the sharp border, we used the latter as a macroscopic reference to identify the crystallographic axes of the surface. As a matter of fact, atomic force microscope images recorded at different magnifications showed that the LW crystallographic direction has a well defined offset angle with respect to the sharp border. Therefore the sample was mounted on the microreflectometer by aligning the incidence plane with the sharp border and than rotating it by the offset angle.

\section{B. Results and discussion}

We apply our theoretical method to understand the reflectance spectra for the sample shown in Fig. 5. We therefore calculate the reduced bands for a close packed fcc crystal with sphere diameter of $222 \mathrm{~nm}$ (fcc lattice constant $a=314 \mathrm{~nm}$ ). We then obtain the reduced DOS and calculate the energy position of the low energy gap and its dependence on $\theta$, as well as the two most important high energy features, i.e., two peaks that are associated with photonic modes. The energy position of the gap is obtained directly from the reduced bands (see Fig. 4), while the position of the peaks is obtained from the DOS shown in Fig. 3. The results are shown in Fig. $6 \mathrm{a}$ and $6 \mathrm{c}$ for the directions LW and LK, respectively. The angular dependence of these energy positions correlates with the dispersion of the photonic bands along the hexagonal surface of the Brillouin zone (Fig. 1b), which

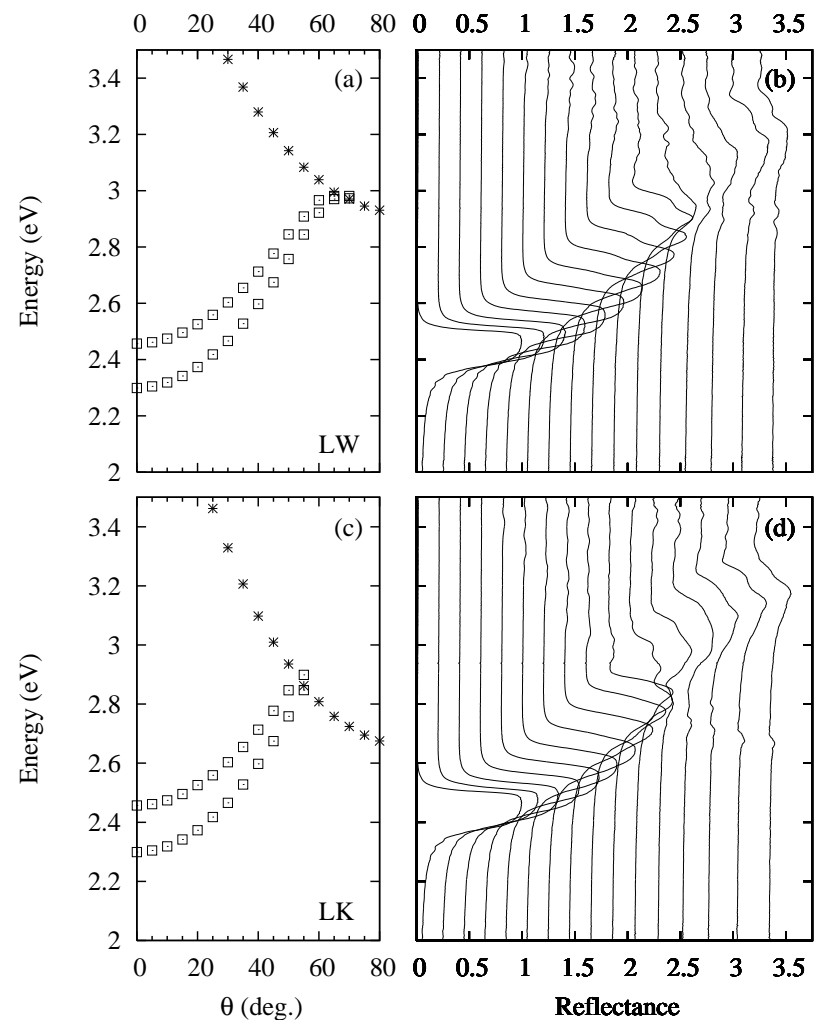

FIG. 6: (ac): Theoretical positions of the gap edges (squares) and of the lowest energy peak (stars) for the LW (a) and the LK (c) direction. The results are for a close-packed fcc structure of polystyrene spheres $(\epsilon=2.53)$ with a lattice constant $a=314 \mathrm{~nm}$. (bd): Experimental reflectance data for several values of $\theta=j \Delta \theta$, with $\Delta \theta=5^{\circ}$ for the LW (b) and the LK (d) direction; curves from left to right correspond to $j=1, \ldots, 16$ and are shifted by the value $0.2(j-1)$.

gives the energies of Van Hove singularities. Notice that the Van Hove singularity for the LK direction has a lower energy (at a given value of the incidence angle) as compared to the LW direction. The Van Hove singularity crosses the band gap at $\theta=70^{\circ}$ for LW and $\theta=60^{\circ}$ for LK. The energies of these structures may be directly compared with the features observed in the reflectance spectra (Fig. 6b and Fig. 6d).

Before starting a comparison of the theoretical and experimental results, we give a description of the latter. Figure $6 \mathrm{~b}$ and $6 \mathrm{~d}$ show the variable-angle micro reflectance spectra of the opal for $\theta$ ranging from $5^{\circ}$ to $80^{\circ}$ in steps of $5^{\circ}$ and along LW and LK, respectively. These spectra are offset horizontally for a more clear view. At near-normal incidence a single, intense (reflectance almost identical to that of the Ag reference mirror), asymmetrical band associated with the stop band is observed at $2.47 \mathrm{eV}$ with a FWHM of about $0.15 \mathrm{eV}$.

When the incidence angle is increased from normal incidence up to $45^{\circ}$, the reflectance maximum shifts towards higher energies slightly reducing its intensity and modifying its shape. This behavior is accounted for by the theoretical band edge positions summarized by the 

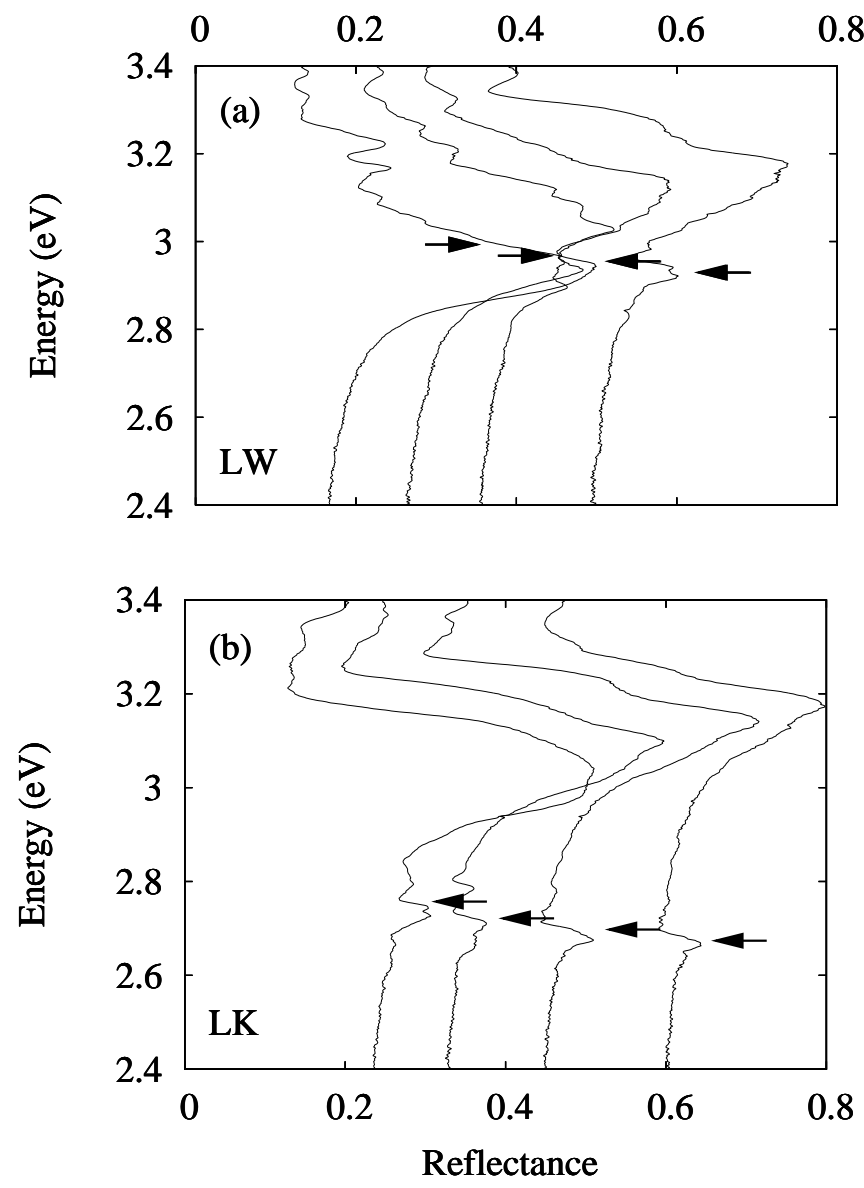

FIG. 7: Enlarged view of experimental reflectance spectra for large incidence angles: $\theta=65^{\circ}, 70^{\circ}, 75^{\circ}, 80^{\circ}$ (from left to right). Arrows indicate the calculated spectral positions of the lower energy Van Hove singularity (Fig. 6a and 6c). Directions are: LW (a) and LK (b).

squares in Fig. 6a (LW) and Fig. 6c (LK). The agreement between calculated and measured gap position as well as its dependence on $\theta$ is very satisfactory.

A more complex situation occurs for higher values of the incidence angle.

We notice that for $\theta \geq 50^{\circ}$ the shape of the spectrum changes in a substantial way and additional features occur. The details of these features depend on the observed direction. To give a better idea of the complicated structure of the spectra recorded for large $\theta$ values and in order to find evidence of the photonic modes, we enlarge the energy scale and show the relevant experimental curves in Fig. 7a (LW) and Fig. 7b (LK). A structured band is observed to regularly shift its maximum in the range from 3 to $3.15 \mathrm{eV}$ retaining the dispersion of the stop band. It may roughly be assigned to the interplay of the stop band and of the excitation of the photonic modes observed in the DOS (Fig. 6a and Fig. 6c), which takes place for large $\theta$. However we also notice the appearence of weak features in the low-energy side of these bands.

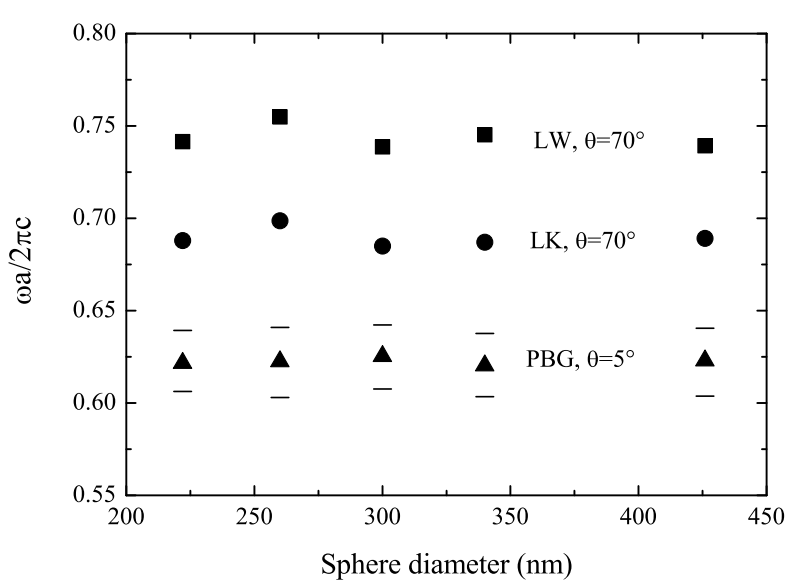

FIG. 8: Scaling of the spectral features, in dimensionless units, as a function of sphere diameter: stop band central position (triangles) and stop band edges (bars) at near-normal incidence; Van Hove singularities at $\theta=70^{\circ}$ for LK (circles) and LW (squares) orientations.

In particular, for the LW direction the weak feature is at about $2.9 \mathrm{eV}$, while for $\mathrm{LK}$ it is at about $2.7 \mathrm{eV}$. When $\theta$ increases, these structures shift towards lower energies, i.e., they behave like the calculated van Hove singularity. Spectral features showing similar dispersions have been previously observed in the transmission spectra of polystyrene ${ }^{54}$ and silica 55 opal films. The low energy calculated peak displayed in Fig. 6a and Fig. 6c for high incidence angles is also reported as an arrow in Fig. 7a and Fig. $7 \mathrm{~b}$ to show a direct comparison with the experimental spectra for each value of $\theta$. We find a very good agreement between the calculated peaks and the weak structures observed in the experimental spectra for both LW and LK directions. Moreover, the possibility of observing a signature of diffraction effects in reflectance spectra has been proved by the calculated spectra shown in Fig.4c. We therefore assign the features having dispersion opposite to the stop band to van Hove singularities in the reduced DOS, i.e., to diffraction from family planes other than those of the (111) one. For large values of the incidence angle along the LW orientation, multiple Bragg scattering close to the $\mathrm{W}$ point takes place and diffraction inside the opal is determined by $\{111\}$ as well as by $\{200\}$ family planes.

In order to further support the assignment of the spectral features observed in $\mathrm{R}$ spectra with van Hove singularities, we checked their scaling with the lattice constant (related to the sphere diameter). Figure 8 shows the experimental spectral position for the stop band maximum and its width at near normal incidence, as well as van Hove singularities measured along the LW and LK directions at $\theta=70^{\circ}$ for opals with different sphere diameters. All these energies, expressed in dimensionless units, are 
clearly independent of the lattice constant thus unambiguously showing that the spectral structures are due to intrinsic properties of the photonic crystal band structure.

It should be remarked that although the presence of flat bands is essential in giving rise to van Hove singularities of the DOS and to diffraction features, the interpretation of the present experiments goes beyond that of Ref 31 in which related observations are attributed only to a higher effective refractive index arising from flat bands and diffraction is not considered. Indeed, when diffraction starts to occur, the optical properties of a photonic crystal cannot be described by a single effective index as several modes propagate in the crystal at the same frequency. The present interpretation does not contradict the multiple Bragg-wave coupling or band branching mechanisms previously introduced ${ }^{24.25 .26 .32}$ and it confirms the identification of multiple diffraction as arising from $\{111\}$ or $\{200\}$ family planes for the different orientations. Extending the previous works, our observations indicate that diffraction-related features in reflectance spectra become increasingly visible for higher values of the angle of incidence: i.e., the onset of diffraction (or, equivalently, the excitation of several photonic modes at the same frequency) is not restricted to the vicinity of the $\mathrm{W}$ or $\mathrm{K} / \mathrm{U}$ points of the Brillouin zone and should be viewed as a more general characteristic of the optical response of the investigated photonic crystals.

We notice that weak spectral features with a negative dispersion as a function of the incidence angle may also be recognized in the range $30^{\circ}-60^{\circ}$ above the band gap peak (Fig. 6b and Fig. 6d). We do not pursue a quantitative analysis of these features, whose identification is not straightforward. In fact photonic modes occuring at relatively high energy are more sensitive to disorder, which may broaden and mix fine spectral features, and also to dispersion of the refractive index. Further work is required in order to establish conclusively the presence of Van Hove singularities at energies above the band gap.

\section{CONCLUSIONS}

In this paper a detailed study of the photonic band structure for artificial opals with close packed fcc structure is reported. The reduced photonic bands and density of states corresponding to a given value of the incidence angle in air are calculated in order to provide a guide for the interpretation of optical spectra. Two main features with opposite dispersions have been identified. A minimum of the reduced DOS corresponds to the stop band in the propagation direction, while maxima and van Hove singularities in the reduced DOS are associated with the excitation of photonic modes in the crystal due to diffraction by higher order planes in the photonic lattice. These modes depend on the investigated orientations of the sample with respect to the normal direction, reflecting the microscopic symmetries of the photonic lattice. By comparing with calculated reflection and diffraction spectra, it has been shown that van Hove singularities do indeed correspond to diffraction peaks and become also visible in reflectance spectra at large values of the incidence angle.

The theoretical data are compared with microreflectance spectra recorded at different incidence angles on bulk artificial opals. There is a very good agreement between theoretical and experimental data for what concerns the dispersion of the stop band. The predicted dispersion of the excited photonic modes is validated by features observed in reflectance spectra for large incidence angles and different sample alignments along the symmetry directions LW and LK, thereby providing evidence for diffraction by higher-order planes (namely $\{111\}$ and $\{200\}$ families), or excitation of photonic modes out of the direction of light propagation. Finally, all the spectral features here discussed and measured in opals grown with different sphere diameter scale with the lattice constant, thus confirming their assignment to intrinsic features of the photonic band structure. The role of light polarization on the optical properties of opaline photonic crystals is currently under investigation.

\section{ACKNOWLEDGMENTS}

The authors are indebted with Mario Agio for providing the scattering-matrix code. They are also grateful to D. Coquillat, J. Galisteo-López and D. Wiersma for helpful conversations. D.C. thanks Prof. G. Dellepiane for her support. This work was partially supported by the Ministry of Education, University and Research (MIUR) through Cofin program.
1 E. Yablonovitch, Phys. Rev. Lett. 58, 2059 (1987).

2 S. John, Phys. Rev. Lett. 58, 2486 (1987).

3 Photonic Crystals and Light Localization in the 21st Century, edited by C.M. Soukoulis, NATO Science Series C, vol. 563 (Kluwer, Dordrecht, 2001).

4 For recent reviews, see e.g. papers in IEEE J. Quantum Electron. 38, Feature Section on Photonic Crystal Structures and Applications, edited by T.F. Krauss and T. Baba, pp. 724-963 (2002).
5 J.E.G.J. Wijnhoven and W.L. Vos, Science 281, 802 (1998).

6 A.A. Zakhidov, R.H. Baughman, Z. Iqbal, C. Cui, I. Khayrullin, S.O. Dantas, J.I. Marti, and V.G. Ralchenko, Science 282, 897 (1998).

7 A. Blanco, E. Chomski, S. Grabtchak, M. Ibisate, S. John, S.W. Leonard, C. López, F. Meseguer, H. Miguez, J.P. Mondia, G.A. Ozin, O. Toader, and H.M. van Driel, Nature 405, 437 (2000). 
8 Yu.A. Vlasov, X.-Z. Bo, J.C. Sturm, and D.J. Norris, Nature 414, 289 (2001).

9 H.S. Sözüer, J.W. Haus, and N. Inguva, Phys. Rev. B 45 13962 (1992).

10 K. Busch, and S. John, Phys. Rev. E 583896 (1998).

${ }^{11}$ W.L. Vos, R. Sprik, A. van Bladeren, A. Imhof, A. Lagendijk, and G.H. Wegdam, Phys. Rev. B 53, 16231 (1996).

12 I.I. Tarhan and G.H. Watson, Phys. Rev. Lett. 76, 315 (1996).

13 Yu.A. Vlasov, V.N. Astratov, O.Z. Karimov, A.A. Kaplyanskii, V.N. Bogomolov, and A.V. Prokofiev, Phys. Rev. B 55, R13357 (1997).

14 V.N. Bogomolov, S.V. Gaponenko, I.N. Germanenko, A.M. Kapitonov, E.P. Petrov, N.V. Gaponenko, A.V. Prokofiev, A.N. Ponyanina, N.I. Silvanovich, and S.M. Samoilovich, Phys. Rev. E 55, 7619 (1997).

15 H. Míguez, C. López, F. Meseguer, A. Blanco, L. Vázquez, R. Mayoral, M. Ocaña, V. Fornés, and A. Mifsud, Appl. Phys. Lett. 71, 1148 (1997).

16 H. Míguez, A. Blanco, F. Meseguer, C. López, H.M. Yates, M.E. Pemble, V. Fornés, and A. Mifsud, Phys. Rev. B 59, 1563 (1999)

17 M. Thijssen, R. Sprik, J.E.G.J. Wijnhoven, M. Megens, T. Narayanan, A. Lagendijk, and W.L. Vos, Phys. Rev. Lett. 83, 2730 (1999).

18 A. Imhof, W.L. Vos, and A. Lagendijk, Phys. Rev. Lett. 83, 2942 (1999).

19 M.M. Megens, J.E.G.J. Wijnhoven, A. Lagendijk, and W.L. Vos, Phys. Rev. A 59, 4727 (1999).

20 A. Reynolds, F. López-Tejeira, D. Cassagne, F.J. GarcíaVidal, C. Jouanin, and J. Sánchez-Dehesa, Phys. Rev. B 60, 11422 (1999).

21 Yu. A. Vlasov, V.N. Astratov, A.V. Baryshev, A.A. Kaplyanskii, O.Z. Karimov, and M.F. Limonov, Phys. Rev. E 61, 5784 (2000).

${ }^{22}$ Yu.A. Vlasov, M. Deutsch, and D.J. Norris, Appl. Phys. Lett. 76, 1627 (2000).

23 H.P. Schriemer, H.M. van Driel, A.F. Koenderink, and W.L. Vos, Phys. Rev. A 63, 011801 (2000).

24 H.M. van Driel and W.L. Vos, Phys. Rev. B 62, 9872 (2000).

25 W.L. Vos and H.M. van Driel, Phys. Lett. A 272, 101 (2000).

26 S.G. Romanov, T. Maka, C.M. Sotomayor Torres, M. Müller, R. Zentel, D. Cassagne, J. Manzanares-Martinez, and C. Jouanin, Phys. Rev. E 63, 056603 (2001).

27 A.F. Koenderink, L. Bechger, H.P. Schriemer, A. Lagendijk, and W.L. Vos, Phys. Rev. Lett. 88, 143903 (2002).

28 J.F.G. Lòpez and W.L. Vos, Phys. Rev. E 66, 036616 (2002).

29 V.N. Astratov, A.M. Adawi, S. Fricker, M.S. Skolnick, D.M. Whittaker, and P.N. Pusey, Phys. Rev. B 66, 165215 (2002).

30 J.F. Galisteo-López, F. López-Tejeira, S. Rubio, C. López, and J. Sánchez-Dehesa, Appl. Phys. Lett. 82, 4068 (2003).

31 H. Míguez, V. Kitaev, and G.A. Ozin, Appl. Phys. Lett. 84, 1239 (2004).

32 J.F. Galisteo-López, E. Palacios-Lidón, E. CastilloMartínez, and C. López, Phys. Rev. B 68, 115109 (2003).

33 J.F. Galisteo-López and C. López, Phys. Rev. B 70, 035108 (2004).

34 V. Yannopapas, N. Stefanou, and A. Modinos, J. Phys: Cond. Matt. 9, 10261 (1997).
35 Z.-Y. Li, and Z.-Q. Zhang, Phys. Rev. B 62, 1516 (2000).

36 V. Yannopapas, N. Stefanou, and A. Modinos, Phys. Rev. Lett. 86, 4811 (2001).

37 T. Ochiai, K. Sakoda, and J. Sánchez-Dehesa, Phys. Rev. B 64, 245113 (2001).

${ }^{38}$ F. López-Tejeira, T. Ochiai, K. Sakoda, and J. SánchezDehesa, Phys. Rev. B 65, 195110 (2002).

39 Z.L. Wang, C.T. Chan, W.Y. Zhang, Z. Chen, N.B. Ming, and P. Sheng, Phys. Rev. E 67, 016612 (2003).

40 N. Eradat, A. Y. Sivachenko, M. E. Raikh, Z. V. Vardeny, A. A. Zakhidov and R. H. Baughman, Appl. Phys. Lett. 80, 3491 (2002).

41 T. Fujita, Y. Sato, T. Kuitani, and T. Ishihara, Phys. Rev. B 57, 12428 (1998).

42 V.N. Astratov, D.M. Whittaker, I.S. Culshaw, R.M. Stevenson, M.S. Skolnick, T.F. Krauss, and R.M. De La Rue, Phys. Rev. B 60, R16255 (1999).

43 V. Pacradouni, W.J. Mandeville, A.R. Cowan, P. Paddon, J.F. Young, and S.R. Johnson, Phys. Rev. B 62, 4204 (2000).

44 M. Galli, M. Agio, L.C. Andreani, L. Atzeni, D. Bajoni, G. Guizzetti, L. Businaro, E. Di Fabrizio, F. Romanato, and A. Passaseo, Eur. Phys. J. B 27, 79 (2002).

45 M. Galli, M. Agio, L.C. Andreani, M. Belotti, G. Guizzetti, F. Marabelli, M. Patrini, P. Bettotti, L. Dal Negro, Z. Gaburro, L. Pavesi, A. Lui, and P. Bellutti, Phys. Rev. B 65, 113111 (2002).

46 D. Comoretto, E. Pavarini, M. Galli, C. Soci, F. Marabelli, and L.C. Andreani, SPIE Proc. 5511, 135 (2004).

47 K.M. Ho, C.T. Chan, and C.M. Soukoulis, Phys. Rev. Lett. 65, (1990) 3152.

48 X. Ma, J.Q. Lu, R.S. Brock, K.M. Jacobs, P. Yang, and X.-H. Hu, Phys. Med. Biol. 48, 4165 (2003).

49 According to Ref. 48, the refractive index $n=1.59$ of polystyrene spheres refers to a wavelength $\lambda=500 \mathrm{~nm}$. Its dispersion has been measured to be no more than $\pm 1.5 \%$ around this value in the wavelength range 400-1600 $\mathrm{nm}$. The effect of the index dispersion is negligibly small in the frequency window relevant for spectral features observed around $2.5-3 \mathrm{eV}$ and discussed in this work.

50 For a given pair of values $(\theta, \phi)$, the wavevector $k_{z}$ satisfying the coupled Eqs. (2), (3) traces a curved path in the fcc Brillouin zone, due to the strongly dispersive properties of the photonic crystal.

51 Notice that if the reduced DOS was summed over both positive and negative $k_{z}$, it would become identical for the LK and LU directions. However, the two orientations are physically inequivalent and give rise to different transmission spectra, thus it is useful to define the reduced DOS by summing over positive $k_{z}$ as done in Eq. (4).

52 D.M. Whittaker and I.S. Culshaw, Phys. Rev. B 60, 2610 (1999).

53 Since the scattering-matrix method of Ref. 52 applies to a structures consisting of patterned layers which are homogeneous along a specified $(z)$ direction, the dielectric spheres in the opal structure have been approximated with cylindrical layers. The results shown in Fig. 4c,d are obtained by averaging over calculations with a number of periods in the [111] direction ranging from 4 to 10, in order to smooth out finite-size oscillations.

54 D. Comoretto, R. Grassi, F. Marabelli, and L.C. Andreani, Mat. Sci Eng. C 23, 61 (2003); D. Comoretto, F. Marabelli, C. Soci, M. Galli, E. Pavarini, M. Patrini and L.C. Andreani, Synt. Met. 139, 633 (2003). 
55 D. Comoretto, D. Cavallo, G. Dellepiane, R. Grassi, F. Marabelli, L.C. Andreani, C.J. Brabec, A. Andreev,

BB10.19.1 (2002). and A.A. Zakhidov, Mat. Res. Soc. Symp. Proc. 708, 